\title{
Urinary Fibrinogen Derivative Excretion and Intraglomerular Fibrin Deposition in Glomerulonephritis
}

\author{
P. NAISH, D. J. EVANS, D. K. PETERS
}

\section{Patients and Methods}

Eighty-one patients with varying diagnoses were studied (see table). The urine from two further patients was also studied. Both had renal tubular disease, one secondary to cadmium poisoning and the other due to congenital oculocerebral-renal syndrome. There was no evidence of glomerular disease in either patient and electrophoresis of their urine showed proteins characteristic of isolated tubular disease. urinary excretion of fibrinogen derivatives (F.D.), and proteinuria were explored in 81 patients with glomerulonephritis. A positive correlation existed between proteinuria and F.D. excretion even when no fibrin could be detected in the glomerulus. In two patients with tubular proteinuria F.D. excretion was also raised, suggesting that tubular reabsorption or catabolism of F.D. or both normally occur.

Disproportionately high titres of F.D. were observed when fibrin was deposited in an extracapillary site, but mesangial fibrin deposition was not accompanied by a higher excretion of F.D. than that observed in patients in whom intraglomerular fibrin was not detected. These observations suggest that the immunofluorescent findings on renal biopsies should be the major criteria on which a trial of anticoagulants in proliferative glomerulonephritis might be instituted and that measurement of urinary F.D. is likely to be of value in monitoring therapy in patients with extracapillary fibrin deposition.

\section{Introduction}

In experimental glomerulonephritis (produced by antibody to glomerular basement membrane) there is a close relation between intraglomerular fibrin deposition, crescent formation, and the development of renal failure (Vassalli and McCluskey, 1964; Naish et al., 1972). In these studies and also in those of Kleinerman (1954) and Halpern et al. (1965) treatment of animals with anticoagulants resulted in a reduction in glomerular damage. In human glomerulonephritis the value of anticoagulants is less clear. This may partly be owing to failure to select the appropriate patients for treatment with anticoagulants. High levels of fibrinogen derivatives (F.D.) have been found in the urine of patients with some types of glomerulonephritis (Naish et al., 1970; Clarkson et al., 1971). This observation suggested that measurement of urinary F.D. might reflect glomerular fibrin deposition and therefore serve as a useful guide to therapy. This suggestion therefore stimulated the present work, which was undertaken to explore systematically the relation between glomerular fibrin deposition and urinary F.D. excretion and to determine the extent to which urinary F.D. excretion is influenced by proteinuria. It was hoped that the results of this study would provide a more rational basis for the use of anticoagulants in glomerulonephritis.

Royal Postgraduate Medical School, London W12 OHS

P. NAISH, M.B., M.R.C.P., Research Fellow; now Consultant Physician, Staffordshire Royal Infirmary

D. J. EVANS, M.B., Lecturer in Pathology

D. K. PETERS, M.B., M.R.C.P., Lecturer in Medicine and Immunology
Diagnosis and Incidence of Intraglomerular Fibrin Deposition In 81 Patients

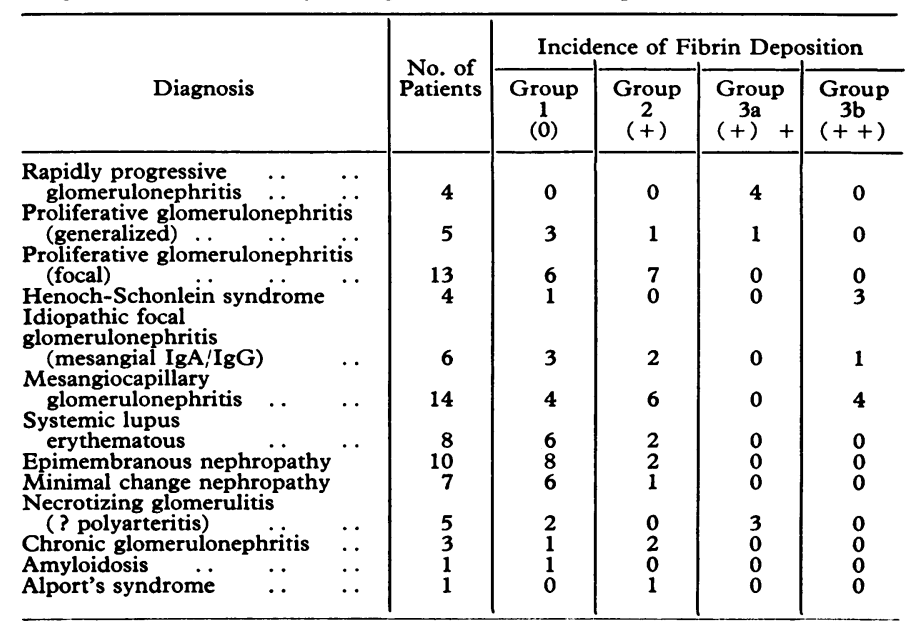

F.D. titre was assayed by the haemagglutination inhibition immunoassay of Merskey et al. (1969) on urine concentrated a hundredfold as previously described (Naish et al., 1973). In our laboratory this system detects $2 \mu \mathrm{g} / \mathrm{ml}$ or more of fibrinogen in urine. Urine samples were obtained from most patients before renal biopsy and from some two weeks or more after biopsy. Results were expressed either as a mean of the titres in three urine samples collected at random on consecutive days or as the titre in a sample pooled from three urine collections obtained on consecutive days. Urine protein concentration was measured by the biuret method before the urine was concentrated.

The renal biopsy specimen was divided into two portions. One was fixed in $15 \%$ formal saline and embedded in paraffin and the other snap-frozen in liquid nitrogen and stored at $-70^{\circ} \mathrm{C}$ if not immediately processed. Sections of $3-4 \mu \mathrm{m}$ were cut from the paraffin block and stained with haematoxylin and eosin, periodic-acid Schiff, and periodic-acid silver methanamine. Frozen tissue was sectioned at $4 \mu \mathrm{m}$ and stained with a monospecific fluoresceinated rabbit antihuman fibrinogen antiserum, prepared in this laboratory. Intraglomerular fibrin deposition was divided into three grades and patients fell into the following three groups according to the amount of fibrin deposited: group 1, no fibrin (grade 0 ); group 2, small amount of fibrin (grade + ); group 3, large amount of fibrin (grade $++)$.

The third group was subdivided into group 3a, when fibrin was deposited in an extracapillary site-that is, in Bowman's space or within crescents-and group $3 b$, when the fibrin was 
present in the mesangium or the parietal areas of glomerular tufts or both. Fibrin deposition was graded independently by two observers (P.N. and D.J.E.) without knowledge of the titre of F.D. in the urine.

\section{Results}

Intraglomerular Fibrin Deposition.-Of the 18 cases of proliferative glomerulonephritis, whether generalized or focal, 9 showed grade + or ++ fibrin deposition. Fibrin was seen in one out of seven cases of minimal change glomerular disease, and in two out of 10 cases of epimembranous nephropathy. All cases of rapidly progressive glomerulonephritis fell into group 3a, with fibrin either in Bowman's space or within crescents. Fibrin deposition in the mesangium or in parietal areas of the

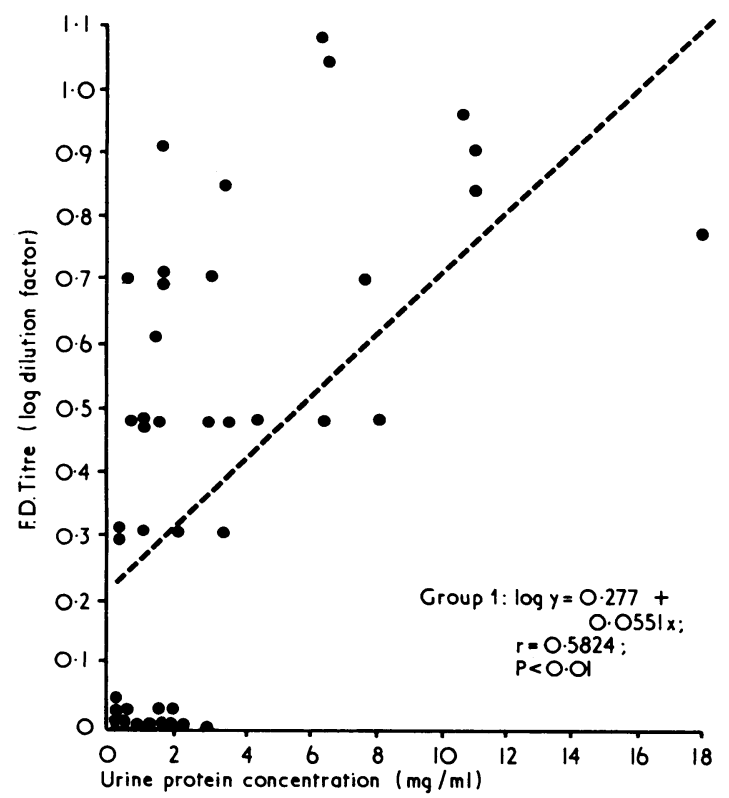

FIG. 1-Relation between proteinuria and urinary F.D. excretion in group 1.

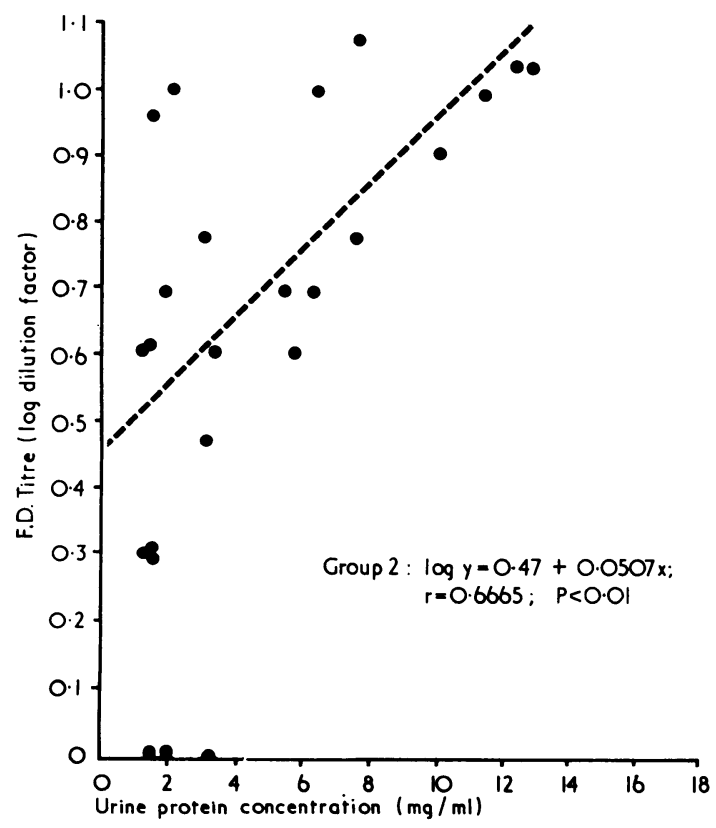

FIG. 2-Relation between proteinuria and urinary F.D. excretion in group 2. glomerular tufts, without occlusion of the capillary lumen (group 3b), was a particular feature of mesangiocapillary nephritis, idiopathic focal nephritis (IgA/IgG disease), and Henoch-Schönlein syndrome (see table).

Urinary F.D. Titres and Proteinuria.-When the patients were classified according to intraglomerular fibrin deposition a significant linear relation existed between F.D. excretion and urine protein concentration in each group except group 3a (figs. 1, 2, and 3). There was no significant difference between the slopes of the regression lines, but there was a significant difference in the intercepts of regression lines between groups 1 and $2(P<0.01)$. This difference was not significant between groups 1 and $3 \mathrm{~b}$. When all the points representing positive intraglomerular fibrin were considered together the relation between F.D. and proteinuria was linear and significant (log $\mathrm{y}=0.452+0.0571 \mathrm{x} ; \mathrm{r}=0.6171 ; \mathrm{P}<0.01)$. The intercept of this regression line was significantly different from that of the group in which no intraglomerular fibrin was detected $(P<0.01)$. F.D. was detected in both samples of urine, concentrated a hundredfold from the two patients with isolated renal tubular disease.

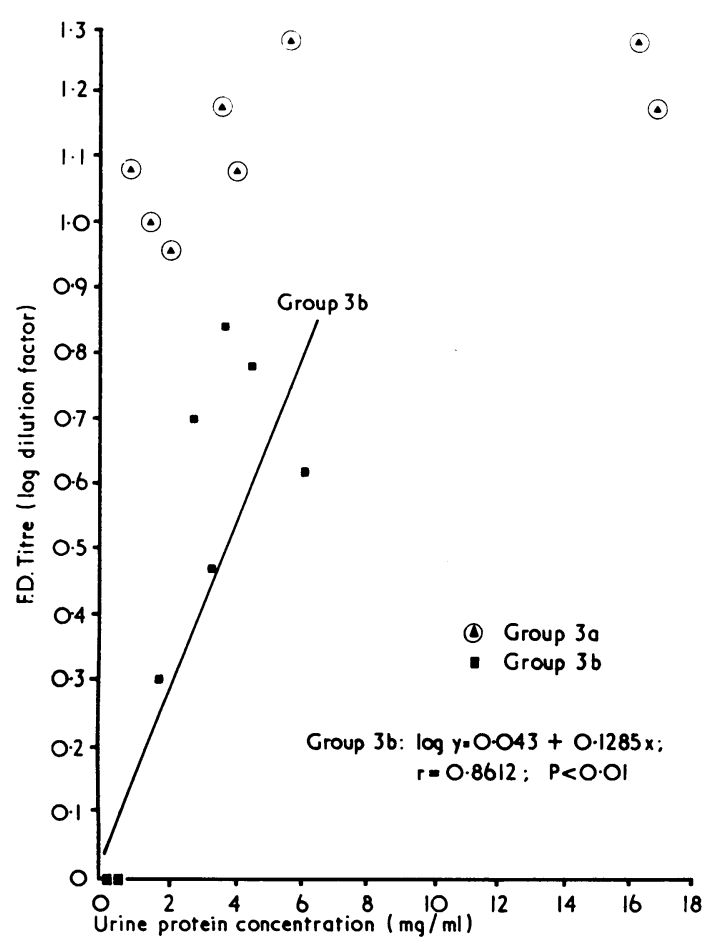

FIG. 3-Relation between proteinuria and urinary F.D. excretion in groups $3 a$ and $3 b$. (Group $3 a: \log y=0.948+$ $0.0208 x ; r=0.4711 ;$ N.S.)

\section{Discussion}

Just over half our patients with proliferative glomerulonephritis showed moderate or large deposits of fibrin in the glomeruli, and all cases of rapidly progressive glomerulonephritis showed large amounts of fibrin outside the glomerular tufts. The results of this part of the study are largely in agreement with those of Morel-Maroger et al. (1972).

F.D. excretion increased with proteinuria. When biopsy specimens were categorized according to fibrin deposition the relation between F.D. excretion and proteinuria persisted in every group except group 3a, and the slopes of these regression lines were not significantly different. Thus even when no fibrin could be detected within glomeruli (group 1) increasing F.D. excretion accompanied increasing proteinuria. These observations suggest that F.D. excretion in the urine, regardless of the amount of intraglomerular fibrin deposition, is partly a non-specific reflection of increased glomerular capillary per- 
meability. There was a significant difference, however, in the intercept of the regression lines of F.D. on proteinuria between groups 1 and 2. Thus F.D. excretion was also related to intraglomerular fibrin deposition. Evidently therefore proper interpretation of the significance of the concentrations of F.D. in urine samples from patients with glomerulonephritis cannot be made without simultaneous measurements of protein concentration. It has previously been assumed that F.D. in urine is solely a reflection of glomerular disease (Clarkson et al., 1971), but the findings in the two patients with isolated renal tubular disease indicate that this may not be so and suggest that under normal circumstances tubular reabsorption or catabolism of F.D. occurs.

There was no significant linear correlation between proteinuria and F.D. titres in group 3a (in which extracapillary fibrin deposition was present). A high level of F.D. excretion was present in each case. This alteration of the relation of F.D. excretion and proteinuria may have been due to the severe glomerular darnage or to the extracapillary site of fibrin deposition or to both these factors. A significant linear relation between F.D. excretion and proteinuria existed, however, when all the patients with intraglomerular fibrin were considered together. Our finding that urinary F.D. excretion was related to proteinuria in all types of proliferative glomerulonephritis except group $3 a$ is at variance with the observations of Clarkson et al. (1971). They found this relation in epimembranous and minimal change nephropathy (in which they assumed there was no intraglomerular fibrin) but failed to show a significant correlation between urine F.D. and proteinuria in their cases of proliferative glomerulonephritis. This may have been owing to their failure to subdivide the cases of proliferative glomerulonephritis according to the site and extent of intraglomerular fibrin deposition.

Comparison of patients in whom large amounts of fibrin were deposited within the glomerular tuft (group 3b) with those in whom fibrin was deposited predominantly in an extracapillary site (group 3a) is of particular interest. Much smaller quantities of F.D. were found in the urine for equivalent amounts of proteinuria in group $3 b$ than in group $3 a$; indeed there was no significant difference in F.D. excretion between group $3 \mathrm{~b}$ and the cases in which no intraglomerular fibrin was detected. Low concentrations of F.D. in the urine were also reported by Davison et al. (1973) in patients with deposits confined to the capillary wall or mesangium. The reasons for the apparent failure of intraglomerular fibrin to appear as F.D. in the urine are uncertain. One possibility may be that products of fibrinogen breakdown in the mesangium do not reach the urinary space. Another is that there may be impairment of intraglomerular mechanisms responsible for breakdown and removal of fibrinogen. If this is so it is interesting to speculate whether failure to clear deposited fibrin is causally related to the development of glomerular disease or is secondary to allergic glomerular damage. Bergstein and Michael (1972) found no correlation between glomerular fibrin deposition and fibrinolytic activity in the renal cortex. The study of glomerular fibrinolytic activity presents problems, however, and in-vitro techniques may not accurately reflect in-vivo events. If indeed failure to clearance of deposited fibrin by whatever mechanism is a feature of these patients then the possibility also arises that this failure is not limited to clearance of deposited fibrin but extends to other macromolecules such as antigen-antibody complexes. If this is so then failure of mesangial cell function could be an important factor in the pathogenesis of some types of glomerulonephritis.

Patients showing the greatest excretion of F.D. were those with fibrin deposition in an extracapillary site. These patients also showed crescent formation as a feature of their histology. This finding is in keeping with our conclusion (Naish et al., 1972) and that of Vassalli and McCluskey (1964; from experimental studies in animals) that intraglomerular fibrin deposition is causally related to crescent formation. Whether extracapillary fibrin deposition results from a qualitatively different kind of glomerular injury or from quantitatively more severe injury or both remains to be resolved. Whatever the correct explanation fibrin in an extracapillary site is almost certainly associated with the histological changes of progressive glomerular disease, and immunofluorescent localization of intraglomerular fibrin would seem to be the best criterion on which to base a trial of anticoagulants in proliferative glomerulonephritis. The measurement of urinary F.D. (related to proteinura) should be of value in assessing the effects of this therapy.

We are grateful to Dr. H. Platt, department of chemical pathology, University College Hospital, London, for the opportunity to study two patients with renal tubular proteinuria. We thank Mrs. R. Ewens for her excellent technical help. P. Naish was supported by a grant from the Medical Research Council.

Requests for reprints should be addressed to Dr. P. Naish, North Staffordshire Royal Infirmary, Stoke-on-Trent.

\section{References} Berger, J. (1969) Transplantation Proceedings, 1, 939.
Bergstein J. M., and Michael, A. F. (1972). Fournal of Laboratory and Clinical

Medicine, 79, 701.
Clarkson, A. R., MacDonald, M. K., Petrie, J. J. B., Cash, J. D., and Robson, J. S. (1971): British Medical fournal, 3, 447.

Davison, A. M., et al. (1973). Fournal of Clinical Pathology, 26, 102.

Halpern, B., Milliez, P., Lagrue, G., Fray, A., and Morard, J. C. (1965). Nature, 205, 257.

Kleinerman, J. (1954). Laboratory Investigation, 3, 495.

Merskey, C., Lalezari, P., and Johnson, A. J. (1969). Proceedings of the Society for Experimental Biology and Medicine, 131, 871.

Morel-Maroger, L., Leatham, A., and Richet, G. (1972). American fournal of Medicine, 53, 170.

Naish, P., Penn, G. B., Evans, D. J., and Peters, D. K. (1972). Clinical

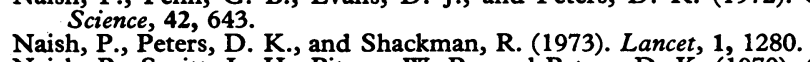

Naish, P., Sevitt, L. H., Pitney, W. R., and Peters, D. K. (1970). Clinical Science, 39, $15 \mathrm{p}$.

Vassalli, P., and McCluskey, R. T. (1964). American fournal of Pathology, 45, 653. 